\title{
ON FINITE COMPLETE PRESENTATIONS AND EXACT DECOMPOSITIONS OF SEMIGROUPS
}

\author{
JOÃO ARAÚJO AND ANTÓNIO MALHEIRO
}

\begin{abstract}
We prove that given a finite (zero) exact right decomposition $(M, T)$ of a semigroup $S$, if $M$ is defined by a finite complete presentation then $S$ is also defined by a finite complete presentation. Exact right decompositions are natural generalizations to semigroups of coset decompositions in groups. As a consequence we deduce that the Zappa-Szép extension of a monoid defined by a finite complete presentation, by a finite monoid is also defined by such a presentation.

It is also shown that when a semigroup $A$ isomorphic to a variant semigroup $A(x)$ that is defined by a finite complete presentation, where $x$ belongs to a sandwich matrix $P$, together with some other conditions, we deduce that the zero Rees matrix semigroup $M^{0}[A ; I, J ; P]$ is also defined by a finite complete presentation.
\end{abstract}

\section{INTRODUCTION}

Let $S$ be a semigroup, $M$ a subsemigroup of $S$ and $T$ a subset of $S$. We say that the pair $(M, T)$ is an exact right decomposition of $S$ if:

(i) $S=M T^{1}$; and

(ii) for every $s \in S$ exists only one $m \in M$ and only one $t \in T^{1}$ such that $s=m t$, where $T^{1}=T \cup\left\{1_{S}\right\}$ and $1_{S}$ is the identity of the monoid $S^{1}$ obtained from $S$ by adding an identity if necessary. This pair origins a decomposition of $S$ as a disjoint union of bijective sets of the form $M t$, with $t \in T^{1}$. As such, exact decompositions of semigroups are obvious generalizations to semigroups of the coset decomposition in groups.

A particular case of the coset decomposition in groups is the concept of exact factorization [16]. Let $G$ be a group and $H, H^{\prime}$ be two subgroups (not necessarily normal); then $\left(H, H^{\prime}\right)$ is said to be an exact factorization of $G$ if $G=H H^{\prime}$ and for every $g \in G$ exists only one $h \in H$ and only one $h^{\prime} \in H^{\prime}$ such that $g=$ $h h^{\prime}$. Obviously when $H$ is normal in $G$ the exact factorization is called semidirect product. Exact factorizations of groups appear under several different names such as general product [20], Zappa-[Rédei-]Szép products [28, 31], and apparently were introduced in 1904 [26].

Date: May 11, 2010.

1991 Mathematics Subject Classification. Primary 68Q42, 20M05 Secondary 03D40, 20M50.

Key words and phrases. Complete rewriting system; complete presentation; zero exact decomposition; factorizable semigroup; transversal; zero Rees matrix semigroup; Zappa-Szép product; semidirect product.

This work was developed within the project POCTI-ISFL-1-143 of CAUL, financed by FCT and FEDER. It is also part of the project "Semigroups and Languages - PTDC/MAT/69514/2006", financed by FCT. 
In the same way, given a semigroup with $M, T<S$, we say that the pair $(M, T)$ is an exact factorization of $S$ if $S=M T$ and for every $s \in S$ exists only one $m \in M$ and only one $t \in T$ such that $s=m t$. Although the concepts of exact decomposition and exact factorization have in general different meanings, it is known that exact factorizations of monoids are also exact decompositions. The fundamental facts about exact factorizations of semigroups were established in [5]. An exact decomposition [factorization] $(M, T)$ is said to be finite if $T$ is finite.

On the other hand finite complete (that is, noetherian and confluent) presentations are used to solve word problems among other algebraic decision problems (see $[4,13,21]$ for examples). The word problem is solved using the "normal form algorithm': given two words $u$ and $v$, we can calculate irreducible elements $u_{0}$ and $v_{0}$ such that $u \stackrel{*}{\rightarrow}_{R} u_{0}$ and $v \stackrel{*}{\rightarrow}_{R} v_{0}$, and we conclude that $u \stackrel{*}{\leftrightarrow}_{R} v$ if and only if $u_{0}$ and $v_{0}$ are identical words. This application reveals the importance of such presentations.

Groves and Smith [9] proved that if $H$ is a subgroup of finite index in $G$ and $H$ is defined by a finite complete presentation, then $G$ is also defined by a finite complete presentation. In [18] the first author generalized this result to some particular cases of factor decompositions of monoids. In particular, it is proved that if a group $G$ is defined by a finite complete presentation then the completely simple semigroup $M[G ; I, J ; P]$ is also defined by a finite complete presentation. These results will be generalized in Section 6 .

As usual in Semigroup Theory and with the advantage of enlarging the class of applications we introduce the notion of zero exact right decomposition for semigroups with zero - see Section 4 for the definition. The main result of the paper is the following theorem which is a direct consequence of Theorem 5.1.

Theorem 1.1. Let $(M, T)$ be a finite (zero) exact decomposition of a semigroup $S$. If $M$ is defined by a finite complete presentation, then $S$ is also defined by a finite complete presentation.

As a consequence we get a result on a natural generalization of the group Zappa[Rédei-]Szép product for the class of semigroups - see [14] for details on Zappa-Szép extensions.

Corollary 1.2. Let $S$ be the Zappa-Szép extension of a monoid $M$ by a finite monoid $T$. Then if $M$ is defined by a finite complete presentation, then $S$ is also defined by a finite complete presentation.

There are more examples of finite (zero) exact decompositions of semigroups such as zero Rees matrix semigroups (see Section 6), or the coset decomposition of groups of finite index, where Theorem 1.1 appears as a natural generalization of Groves and Smith result [9]. The consideration of the converse of Theorem 1.1 immediately suggests the following problem:

Open question 1.3. Given a finite (zero) exact decomposition $(M, T)$ of a semigroup (with zero) $S$ defined by a finite complete presentation, is $M$ also defined by such a presentation?

A positive answer to this question would necessarily solve the analogous hard open problem for groups, where it is asked whether the property of being defined by a finite complete presentation is preserved on passing from a group to a finite index subgroup - see [9] and also the partial solution in [24]. 
It is known that a monoid defined by a finite complete presentation is of type $F P_{n}$, for all $n \in \mathbb{N}[1]$ and also of finite derivation type (FDT for short) [27]. The two properties $F P_{3}$ and $F D T$ are equivalent in the class of groups [6] (see also [22]). Also, the property $F P_{3}$ is preserved when passing from a group to some finite index subgroup, and vice-versa. So it is natural to ask the following:

Open question 1.4. Given a finite (zero) exact decomposition $(M, T)$ of a semigroup (with zero) $S$, does the property of having FDT hold in $S$ if and only if it holds in $M$ ?

Partial solutions to this open problem can be found in [19] and [30].

\section{Preliminaries}

Let $X$ be an alphabet. We denote by $X^{*}$ the free monoid on $X$ and by $X^{+}$the free semigroup on $X$. the empty word on $X^{*}$ will be denoted by 1 . For an element of $X^{*}$, a word $w$, we denote the length of $w$ by $|w|$. We use $|w|_{Y}$ to denote the number of occurrences of letters from the subset $Y$ of $X$ in the word $w \in X^{*}$.

A presentation is a pair $\langle X \mid R\rangle$, where $X$ is an alphabet and $R$ is a binary relation in $X^{*}$. The set $R$ is also referred to as rewriting system and its elements as rewriting rules. Usually, a rewriting rule $r \in R$ is written in the form $r=\left(r_{+1}, r_{-1}\right)$ or, simply, $r_{+1} \rightarrow r_{-1}$. We say that the presentation is finite if both $R$ and $X$ are finite.

In $X^{*}$ we define a binary relation, $\rightarrow_{R}$, denoted as single-step reduction, in the following way:

$$
u \rightarrow_{R} v \Leftrightarrow u=w_{1} r_{+1} w_{2} \text { and } v=w_{1} r_{-1} w_{2}
$$

for some $\left(r_{+1}, r_{-1}\right) \in R$ and $w_{1}, w_{2} \in X^{*}$. The transitive and reflexive closure of $\rightarrow_{R}$ is denoted by $\stackrel{*}{\longrightarrow}_{R}$. A word $u \in X^{*}$ is said to be $R$-reducible, if there is a word $v \in X^{*}$ such that $u \rightarrow_{R} v$. If a word is not $R$-reducible, it is called $R$-irreducible or simply irreducible. By $\operatorname{Irr}(R)$ we denote the set of all $R$-irreducible words.

By $\stackrel{*}{\leftrightarrow}_{R}$ we denote the equivalence relation induced by $\rightarrow_{R}$ which is a congruence on the free monoid $X^{*}$. The quotient of the free monoid $X^{*}$ by what is called the Thue congruence $\stackrel{*}{\leftrightarrow}_{R}$ generated by $R$ origins the monoid defined by $R$ and it is denoted by $M(X ; R)$. The set $X$ is called the generating set and $R$ the set of defining relations. More generally, a monoid is said to be defined by the presentation $\langle X \mid R\rangle$ or by the rewriting system $R$ if $M \cong M(X ; R)$. Thus, the elements of $M$ are identified with congruence classes of words from $X^{*}$. We will sometimes identify words and elements they represent, writing $u=v$ if the two words $u, v \in X^{*}$ represent the same element of $M$. We shall write $u \equiv v$ if they are identical as words.

We say that a rewriting system $R$ on $X$ is noetherian if the relation $\rightarrow_{R}$ is wellfounded, in other words, if there are no infinite descending chains

$$
w_{1} \rightarrow_{R} w_{2} \rightarrow_{R} w_{3} \rightarrow_{R} \cdots \rightarrow_{R} w_{n} \rightarrow_{R} \cdots .
$$

We say that $R$ is confluent if whenever we have $u \stackrel{*}{\longrightarrow} v$ and $u \stackrel{*}{\longrightarrow}_{R} v^{\prime}$ there is a word $w \in X^{*}$ such that $v \stackrel{*}{\longrightarrow} w$ and $v^{\prime} \stackrel{*}{\longrightarrow}_{R} w$. If $R$ is simultaneously noetherian and confluent we say that $R$ is complete. We say that a presentation is noetherian, confluent or complete if its associated rewriting system has the respective property. 
It is easy to verify that, if $R$ is a noetherian rewriting system, each congruence class of $M(X ; R)$ contains at least one irreducible element. Assuming $R$ noetherian, then $R$ is a complete rewriting system if and only if each congruence class of $M(X ; R)$ contains exactly one irreducible element [29, Theorem 1.2.2]. Hence, a complete rewriting system fixes a unique normal form for each of its congruence classes.

In the above concepts, if we replace monoid by semigroup and $X^{*}$ by $X^{+}$we get similar definitions and the analogous results also hold. It is straightforward to prove that a monoid is defined by a finite complete monoid presentation if and only if it is defined by a finite semigroup presentation - see [8] for details. Hence the results stated in this paper do not specify the kind of presentation being used since there is no ambiguity doing so.

It is important to notice that for a finite rewriting system $R$ on a set $X$ each word $u$ of $X^{*}$ can only be reduced in finitely many ways. If we also assume that $R$ is noetherian then each descending chain starting at $u$ has finite length and thus there exists a maximum on the length of any word appearing on all descending chains starting at $u$. That maximum is called the stretch of $u$ and it is denoted by $s t_{R}(u)$. Observe that if $u \rightarrow_{R} v$ then $s t_{R}(u) \geq s t_{R}(v)$. Also note that if $v$ is a proper factor of $u$ then $s t_{R}(u)>s t_{R}(v)$.

We end this section with a technical result that gives sufficient conditions on a rewriting systems to be noetherian.

Proposition 2.1. Let $R$ be a finite noetherian rewriting system on the alphabet $X$. Let $Y$ be an alphabet disjoint from $X$. Then a rewriting system on $X \cup Y$ consisting of rewriting rules from $R$ or of the form $l \rightarrow r$, with

- $|l|_{Y}>|r|_{Y} ;$ or

- $(l, r) \in X^{*} Y X^{+} \times X^{*} Y$; or

- $(l, r) \in X^{+} Y \times Y$,

is noetherian.

Proof. Let $R^{\prime}$ be a rewriting system on the conditions of the statement. We shall prove that $R^{\prime}$ is noetherian. In order to do that we introduce a binary relation $\succ$ on $(X \cup Y)^{+}$which can be easily checked to be irreflexive, transitive and well-founded.

A word $w$ in $(X \cup Y)^{*}$ has the form $x_{n} u_{n} x_{n-1} \cdots x_{1} u_{1} x_{0}$, with $n \in \mathbb{N}_{0}, u_{i} \in Y$, and $x_{j} \in X^{*}$. In the same way a word $w^{\prime} \in(X \cup Y)^{*}$ has the form $x_{m}^{\prime} u_{m}^{\prime} x_{m-1}^{\prime} \cdots x_{1}^{\prime} u_{1}^{\prime} x_{0}^{\prime}$. We write $w \succ w^{\prime}$ if:

(1) $n>m$; or

(2) $n=m$ and exists $k \in\{0, \ldots, n\}$ such that $x_{i} \equiv x_{i}^{\prime}$, for $i<k$, and $s t_{R}\left(x_{k}\right)>$ $s t_{R}\left(x_{k}^{\prime}\right) ;$ or

(3) $n=m$ and exists $k \in\{0, \ldots, n\}$ such that $x_{i} \equiv x_{i}^{\prime}$, for $i<k, s t_{R}\left(x_{k}\right)=$ $s t_{R}\left(x_{k}^{\prime}\right)$ and $x_{k} \rightarrow_{R} x_{k}^{\prime}$

It is now routine to check that, for any words $w, w^{\prime} \in(X \cup Y)^{*}$, if $w \rightarrow R^{\prime} w^{\prime}$ then $w \succ w^{\prime}$ and thus $R^{\prime}$ is noetherian since $\succ$ is well-founded. Nevertheless, it is worth observing that for some of the relations of $R^{\prime}$ we will get $x_{k}^{\prime}$ as a proper factor of $x_{k}$, for some $k$, and hence the stretch of the first is strictly less than the stretch of the second.

Further information on rewriting systems can be found in [4]. 


\section{REWRITING SYSTEMS ON SEMIGROUPS WITH ZERO}

A semigroup with zero presentation is a pair $\langle X \mid R\rangle$ where $R$ is a rewriting system on the alphabet $X \cup\{0\}$ being 0 a symbol not in $X$. This pair origins a semigroup with zero in the following way: we add to the rewriting system $R$ the rewriting rules $(0 x, 0)$ and $(x 0,0)$, for any $x \in X$, and the rewriting rule $(00,0)$; with this new rewriting system, say $R_{0}$, we get the Thue congruence $\stackrel{*}{\leftrightarrow} R_{0}$ and hence the semigroup resulting from the quotient of the free semigroup $(X \cup\{0\})^{+}$by $\stackrel{*}{\leftrightarrow}_{R_{0}}$ Clearly the congruence class of the word 0 is a zero on the semigroup. This semigroup with zero is denoted by $S_{0}(X ; R)$.

We say that a semigroup with zero $S$ is defined by the semigroup with zero presentation $\langle X \mid R\rangle$ if $S$ is isomorphic to $S_{0}(X ; R)$. Also, we say that $\langle X \mid R\rangle$ is a semigroup with zero presentation that defines $S$.

One of the easiest ways to obtain a semigroup with zero is to add to a semigroup an extra element which will behave like a zero. Let us consider the following semigroup construction. Let $S$ be a semigroup (with or without a zero) and let 0 be a symbol not in $S$. Consider the set $S \cup\{0\}$ which will be denoted by $S^{0}$. On this set we define a binary operation - by extending the binary operation on $S$ to $S^{0}$ defining $0 \cdot s=s \cdot 0=0$, for any $s \in S^{0}$. This set $S^{0}$ equipped with this new operation is a semigroup with zero. Notice that even if $S$ has originally a zero element the new semigroup $S^{0}$ is a semigroup with a zero distinct from the original zero of $S$.

Proposition 3.1. Let $R$ be a finite complete rewriting system on the alphabet $X$. Let 0 be a symbol not in $X$. Then the rewriting system

$$
R \cup\{(0 x, 0),(x 0,0): x \in X \cup\{0\}\}
$$

on the alphabet $X \cup\{0\}$ is complete. In particular, if $S$ is defined by a finite complete rewriting system then so does the semigroup $S^{0}$ obtained from $S$ by adding a zero element.

Proof. Let $S$ be the semigroup defined by the finite complete presentation $\langle X \mid R\rangle$. If in the Proposition 2.1 we take $Y$ to be the set $\{0\}$ we can conclude that the rewriting system $R_{0}=R \cup\{(0 x, 0),(x 0,0): x \in X \cup\{0\}\}$, is noetherian.

Clearly, the semigroup presentation $\left\langle X \cup\{0\} \mid R_{0}\right\rangle$ defines the semigroup with zero $S^{0}$. Also, the irreducible elements of the rewriting system $R_{0}$ are in one-to-one correspondence with $S^{0}$. Therefore the rewriting system $R_{0}$ is complete.

Let $S$ be a semigroup with zero defined by the [finite] complete semigroup presentation $\langle X \mid R\rangle$. Suppose that the zero of $S$ is represented by some word $z$ on $X^{+}$. Applying a Tietze transformation we get a semigroup presentation $\langle X \cup\{0\} \mid R \cup\{(z, 0)\}\rangle$ which also defines the semigroup $S$. In fact if 0 is a symbol not in $X$ then the rewriting system on $X \cup\{0\}$,

$$
R \cup\{(z, 0)\} \cup\{(0 x, 0),(x 0,0): x \in X \cup\{0\}\}
$$

is [finite] complete and defines $S$, being 0 the irreducible representing the zero of $S$ [7, Proposition 5].

The next example shows that the analysis of the completeness of a semigroup with zero presentation should involve the rewriting rules that are implicit in the semigroup with zero presentation. 
Example 3.2. Let us consider the semigroup with zero defined by the complete semigroup presentation $\left\langle a \mid a^{3}=a^{2}\right\rangle$. This is a two element semigroup, composed by the congruence classes of the irreducible words a and $a^{2}$. It is easy to see that the semigroup with zero presentation $\left\langle a \mid a^{3}=a^{2}, a^{2}=0\right\rangle$ is noetherian but both irreducible words $a 0$ and 0 represent the zero of $S$. Thus the rewriting system is not complete.

By [7, Proposition 5], if we add to the rewriting system the implicit rewriting rules of a semigroup with zero presentation we get a complete rewriting system.

Taking into account the previous considerations, a semigroup with zero presentation $\langle X \mid R\rangle$ will be said complete if the semigroup presentation

$$
\langle X \cup\{0\} \mid R, 0 x \rightarrow 0, x 0 \rightarrow 0,(x \in X \cup\{0\})\rangle
$$

is complete.

By Proposition 3.1, if $\langle X \mid R\rangle$ is a complete semigroup presentation defining $S$ then $\langle X \mid R\rangle$ is a complete semigroup with zero presentation defining $S^{0}$ where 0 is the irreducible representative of the zero of $S^{0}$.

Proposition 3.3. Every [finite] complete semigroup (with zero) presentation defining a semigroup with zero $S$ induces a [finite] complete semigroup with zero presentation defining $S$ where the letter 0 is the irreducible element representing the zero of $S$.

Proof. If $S$ is a semigroup with zero defined by the complete semigroup presentation $\langle X \mid R\rangle$ then by [7, Proposition 5$]\langle X \mid R, z \rightarrow 0\rangle$ is a complete semigroup with zero presentation defining $S$ where the letter 0 is the irreducible element representing the zero of $S$.

If $S$ is defined by the complete semigroup with zero presentation $\langle X \mid R\rangle$, where 0 is not irreducible, it means that $\langle X \cup\{0\} \mid R, 0 x \rightarrow 0, x 0 \rightarrow 0,(x \in X \cup\{0\})\rangle$ is a complete semigroup presentation defining $S$. Now, for technical reasons let us replace the letter 0 by another letter not in $X \cup\{0\}$. After that and arguing as in the last paragraph we get a complete semigroup with zero presentation defining $S$ where the letter 0 is the irreducible element representing the zero of $S$.

\section{EXACT AND ZERO EXACT DECOMPOSITIONS}

In this section we present the basic definitions and results on zero exact decompositions.

Let $(M, T)$ be an exact decomposition of a semigroup $S$. If the semigroup $S$ has a zero the notion of exact right decomposition imposes several restrictions to the semigroups under consideration. If the zero is an element of $T$ or of $M$ we easily conclude that at least one of them has only one element. Otherwise, since the zero must be a product of an element in $M$ by an element in $T$, and $M$ is a subsemigroup and using the uniqueness of the decomposition we conclude that $M$ is a right zero semigroup. In view of the previous comments the definition of exact decomposition is modified and it is introduced the notion of zero exact right decomposition of a semigroup with zero.

Let $S$ be a semigroup with zero denoted by 0 , let $M$ be a subsemigroup of $S$ and $T$ be a subset of $S$. The pair $(M, T)$ is said to be a zero exact right decomposition of $S$ if:

(i) $S \backslash\{0\}=(M \backslash\{0\}) T$; and 
(ii) for every non-zero element $s$ of $S$ exists only one $m \in M \backslash\{0\}$ and only one $t \in T^{1}$ such that $s=m t$.

As mentioned in the introduction $T^{1}$ represents the set $T \cup\left\{1_{S}\right\}$, where $1_{S}$ is the identity of the monoid $S^{1}$. If such pair exists the semigroup $S$ is said to be zero exact right decomposable.

Lemma 4.1. Let $(M, T)$ be a zero exact right decomposition of a semigroup $S$. We have

(i) $t \in(M \backslash\{0\}) t$, for any $t \in T \backslash\left\{1_{S}\right\}$;

(ii) $S=\{0\} \cup\left(\dot{\cup}_{t \in T}(M \backslash\{0\}) t\right)$;

Proof. (i) Let $t \in T \backslash\left\{1_{S}\right\} \subseteq S \backslash\{0\}$. Then $t=m t^{\prime}$, for some $m \in M \backslash\{0\}$, and $t^{\prime} \in T$. Hence we have the equality $m t=m^{2} t^{\prime}$, with $m, m^{2} \in M \backslash\{0\}$ and $t, t^{\prime} \in T$. By the uniqueness property we get $t=t^{\prime}$. From these arguments we conclude that $t \in(M \backslash\{0\}) t$.

(ii) Obvious from the previous item.

Remark 4.2. The previous lemma also holds for the non zero case, in which case it is enough to remove the zero from the statements.

Regarding the above lemma and the notion of coset decomposition for groups, the set $T$ will be called a right transversal for $M$ in $S$. Throughout the paper we will consider right transversals so by transversal we shall always mean a right transversal and by a (zero) exact decomposition a zero exact right decomposition.

Emphasizing the fact that the transversal will always include the identity of $S^{1}$ we will write $T^{1}$ to represent the transversal and $T$ will be the set such that $T^{1}=T \dot{\cup}\{1\}$. Notice also that if $(M, T)$ is a (zero) exact decomposition of a monoid $S$, then the identity of $S$ also belongs to $M$.

On the above definition notice that $M$ may or may not include the zero of $S$. If it does not include we can always consider the subsemigroup with zero $M \cup\{0\}$. Recall that if $M$ is defined by a complete presentation then, by Proposition 3.1, also $M \cup\{0\} \cong M^{0}$ is defined by a complete presentation.

\section{Proof of the main theorem}

In this section we present a theorem in which a finite complete presentation of a zero exact decomposable semigroup $S$ is given, provided the decomposition has a finite transversal and the subsemigroup is defined by a finite complete presentation. As a consequence we get the main result of the paper Theorem 1.1.

Theorem 5.1. Let $(M, T)$ be a zero right decomposition of a semigroup with zero $S$. Suppose that $M$ contains the zero of $S$ and that it is defined by the finite complete semigroup with zero presentation $\langle X \mid R\rangle$, where 0 is irreducible. Then the semigroup with zero presentation with generators $X \cup T$ and rewriting rules

$$
\begin{aligned}
& \left(\mathrm{R}_{1}\right) \quad t_{1} t_{2} \rightarrow\left\{\begin{array}{ll}
m t_{3}, & t_{1} t_{2}={ }_{S} m t_{3} ; \\
0, & t_{1} t_{2}={ }_{S} 0 .
\end{array} \quad\left(t_{1}, t_{2} \in T, t_{3} \in T \cup\{1\}, m \in \operatorname{Irr}(R)\right)\right. \\
& \left(\mathrm{R}_{2}\right) \quad t_{1} x \rightarrow\left\{\begin{array}{ll}
m t_{2}, & t_{1} x={ }_{S} m t_{2} ; \\
0, & t_{1} x={ }_{S} 0 .
\end{array} \quad\left(t_{1} \in T, t_{2} \in T \cup\{1\}, x \in X, m \in \operatorname{Irr}(R)\right)\right. \\
& \left(\mathrm{R}_{3}\right) \quad m t \rightarrow t \quad\left(t \in T, m \in \operatorname{Irr}(R), m t={ }_{S} t\right)
\end{aligned}
$$

is [finite] complete and defines $S$. 
Proof. Denote by $R^{\prime}$ the set $R \cup R_{1} \cup R_{2} \cup R_{3} \cup R_{4}$, where $R_{4}$ are the rewriting rules of the form $0 y \rightarrow 0, y 0 \rightarrow 0$, for all $y \in X \cup\{0\} \cup T$. Notice that if $T$ is finite and $\langle X \mid R\rangle$ is finite then $R^{\prime}$ is also finite.

From the conditions of the theorem we know that the semigroup presentation $\langle X \cup\{0\} \mid R, 0 x \rightarrow 0, x 0 \rightarrow 0,(x \in X \cup\{0\})\rangle$ is complete and defines $M$. The Proposition 2.1 can be applied considering the disjoint sets $X \cup\{0\}$ and $T$. Indeed, it is straightforward to check that each rewriting rule from $R^{\prime}$ satisfies at least one of the possibilities given in the conditions of the proposition. Therefore $R^{\prime}$ is noetherian.

To conclude our proof it is now sufficient to show that the relations from $R^{\prime}$ hold in $S$ and that the elements of the semigroup $S$ are in one-to-one correspondence with the irreducible elements of the new presentation. We shall identify the elements of $(X \cup\{0\} \cup T)^{+}$with the corresponding elements of $S$ by identifying an element of $X^{+}$with the corresponding representative in $M$ and any letter of $T$ with the correspondent element in $S$ of $T$.

Since each non-zero element of $S$ can be decomposed in the form $m t$, with $m \in M \backslash\{0\}$ and $t \in T^{1}$, it is clear from their definition that the relations in $R_{1}$ and $R_{2}$ hold in $S$. Recall that in $S$ some of the products might be zero. Hence, in some of the rewriting rules we will have to choose between the appropriate decomposition of the form $m t$ or the zero of $S$. By Lemma 4.1 each $t \in T$ belongs to $M t$, and hence for each $t \in T$ there exists an $R$-irreducible word $m_{t}$ such that $m_{t} t=t$. Thus all relations from $R_{3}$ hold in $S$.

For each $t \in T$ let $m_{t}$ denote the irreducible element of $R$ such that $m_{t} t=t$. We claim that the set of irreducible elements of the rewriting system $R^{\prime}$ on the alphabet $X \cup\{0\} \cup T$ is $\bigcup_{t \in T}\left(\operatorname{Irr}(R) \backslash\left\{m_{t}\right\}\right) t \cup T \cup \operatorname{Irr}(R) \cup\{0\}$. First observe that all words in this set are irreducible. Now, the process to obtain an irreducible element goes as follows: if the word contains the letter 0 then using relations from $R_{4}$ we get the irreducible word 0 ; otherwise, using rewriting rules from $R_{2}$ we can obtain a word with no letters from $T$ on the left of a letter from $X$; then, using the relations from $R_{1}$ and from $R$ we get an $R$-irreducible element on the left of a letter (it might be the empty word) from $T^{1}$; in case we get $m_{t} t$, for some $t \in T$, we apply a rewriting rule from $R_{3}$ to obtain a letter in $T$.

As already mentioned each non-zero element of $S$ is uniquely decomposable as the product of an element of $M \backslash\{0\}$ and an element of $T^{1}$. Clearly the set $(M \backslash\{0\}) T=$ $S \backslash M$ is in one-to-one correspondence with $\bigcup_{t \in T}\left(\operatorname{Irr}(R) \backslash\left\{m_{t}\right\}\right) t \cup T$ and $M$ is in one-to-one correspondence with $\operatorname{Irr}(R)$. Thus we conclude that $S$ is one-to-one correspondence with our set of irreducible elements of $R^{\prime}$ as required.

Therefore, the [finite] semigroup with zero presentation in the theorem is complete and defines the semigroup $S$.

Proof of Theorem 1.1. By Proposition 3.3 we can obtain from any [finite] complete semigroup presentation a [finite] complete semigroup with zero presentation defining the same monoid where 0 is irreducible. Hence the case of semigroups with zero in Theorem 1.1 follows from the previous theorem.

The non-zero case can be obtained in the following way. Suppose that $(M, T)$ is an exact right decomposition of a semigroup without a zero $S$. Then $\left(M^{0}, T\right)$ becomes a zero exact right decomposition of $S^{0}$. By Proposition 3.1 the semigroup $M^{0}$ is defined by a finite complete presentation since $M$ also is. Therefore, applying 
the result for semigroups with zero we conclude that $S^{0}$ is defined by a [finite] complete presentation.

Observe that $S$ is a subsemigroup of $S^{0}$ whose complement is an ideal. Hence, by $[23$, Theorem $\mathrm{C}]$ we deduce that $S$ is defined by a [finite] complete presentation.

\section{Applications to zero Rees matrix semigroups}

Completely (0-)simple semigroups are an important class of semigroups. The problem of relating the properties of the semigroup with the properties of its maximal subgroups was studied in several papers (see for example $[2,3,12,15,17$, $18,19])$. They can be defined using the notion of ideal. A non-empty set $A$ of a semigroup $S$ is called a left ideal if $S A \subseteq A$, a right ideal if $A S \subseteq A$ and an ideal if it is both a left and a right ideal. Completely (0-)simple semigroups, among other characterizations (see [11]), are those semigroups with no proper ideals, except $\{0\}$ for completely 0-simple, and with (0-)minimal left and right ideals.

There is a particular semigroup construction first used by Rees [25] to obtain a description of the completely simple and completely 0 -simple semigroups in terms of their maximal subgroups. This construction can be generalized replacing the subgroups by a general semigroup $A$ and it is usually called (zero) Rees matrix semigroup over $A$.

The zero Rees matrix semigroups can be defined in the following terms: let $A$ be a semigroup, let $I$ and $J$ be non-empty sets and let $P=\left(p_{j i}\right)$ be a $J \times I$ matrix with entries in $A \cup\{0\}$; suppose that $P$ is regular, that is, no row or column consists entirely of zeros; the set $I \times A \times J \cup\{0\}$ with multiplication

$$
\left(i_{1}, a_{1}, j_{1}\right)\left(i_{2}, a_{2}, j_{2}\right)= \begin{cases}\left(i_{1}, a_{1} p_{j_{1} i_{2}} a_{2}, j_{2}\right), & \text { if } p_{j_{1} i_{2}} \in A \\ 0, & \text { if } p_{j_{1} i_{2}}=0\end{cases}
$$

is called the $I \times J$ zero Rees matrix semigroup over the semigroup $A$ with the sandwich matrix $P$ and it is denoted by $M^{0}[A ; I, J ; P]$. The Rees Theorem states that if $A$ is a group we get a completely 0 -simple semigroup, and that every completely 0 -simple semigroup is isomorphic to a semigroup constructed in this way.

If in the above definition we allow $P$ to have only elements from $A$ and the set of elements is $I \times A \times J$ we get the definition of $I \times J$ Rees matrix semigroup over the semigroup $A$ with sandwich matrix $P$ usually denoted by $M[A ; I, J ; P]$.

Let $A$ be a semigroup and let $x \in A$. We denote by $A(x)$ the semigroup, known as the variant of $A$, with base set $A$ and multiplication $*$ given by $a * b=a x b$, for all $a, b \in A$. See [10] for more on variant semigroups.

Before stating the main result of the section let us recall that the Rees matrix semigroup $M[A ; I, J ; P]$ is finitely presented if and only if $A$ is finitely presented and the sets $I, J$ and $S \backslash U$ are finite, where $U$ is the ideal of $S$ generated by the entries of $P[3]$.

Theorem 6.1. Let $A$ be a semigroup, let $I$ and $J$ be non-empty finite sets and let $P$ be a regular $J \times I$ matrix with entries in $A(A \dot{\cup}\{0\})$. Suppose that there exists an entry $x$ of $P$ and $y, z \in A$ such that:

(i) $A \cong A(x)$;

(ii) the inner right translation $\rho_{x y}: A \rightarrow A, a \mapsto$ axy, is bijective;

(iii) the inner left translation $\lambda_{z x}: A \rightarrow A, a \mapsto z x a$, is bijective. 
If $A$ is defined by a finite complete presentation then the (zero) Rees matrix semigroup $M[A ; I, J ; P]\left(M^{0}[A ; I, J ; P]\right)$ is also defined by a finite complete presentation.

Proof. Let us first prove for the zero Rees matrix semigroup. Without loss of generality suppose that $1 \in I, 1 \in J$ and $p_{11}=x$. In addition suppose $A$ is defined by a finite complete presentation.

Clearly the sets $\{1\} \times A \times\{1\},\{1\} \times A \times\{1\} \cup\{0\}$ and $\{1\} \times A \times J \cup\{0\}$ are subsemigroups of the zero Rees matrix semigroup $M^{0}[A ; I, J ; P]$ and by condition (i) the semigroups $\{1\} \times A \times\{1\}$ and $A$ are isomorphic. Therefore, by assumption and by Proposition 3.1 also $\{1\} \times A \times\{1\} \cup\{0\}$ is defined by a finite complete presentation.

We claim that the pair $\left(\{1\} \times A \times\{1\} \cup\{0\},\left(\{1\} \times\{y\} \times J^{\prime}\right)^{1}\right)$ is a finite zero exact right decomposition of $\{1\} \times A \times J \cup\{0\}$, where $J^{\prime}$ is the set $J \backslash\{1\}$. Indeed, since $\rho_{x y}$ is bijective, each non-zero element $(1, a, j)$ of $\{1\} \times A \times J \cup\{0\}$ is uniquely decomposed as $\left(1, a^{\prime}, 1\right)(1, y, j)$, for some $a^{\prime} \in A$ such that $a^{\prime} x y=a$. Consequently, by Theorem 1.1 the semigroup $\{1\} \times A \times J \cup\{0\}$ is defined by a finite complete presentation.

Denote by $I^{\prime}$ the set $I \backslash\{1\}$. By symmetry it can be proved that $\left(\{1\} \times A \times J \cup\{0\},\left(I^{\prime} \times\{z\} \times\{1\}\right)^{1}\right)$ is a finite zero exact left decomposition of the zero Rees matrix semigroup $M^{0}[A ; I, J ; P]$. Thus applying the dual of Theorem 1.1 for left transversals we get the intended result.

For the non-zero case the matrix $P$ has only non-zero elements and hence $M[A ; I, J ; P]$ is a subsemigroup of $M^{0}[A ; I, J ; P]$ whose complement is an ideal. Therefore, by $[23$, Theorem $\mathrm{C}]$ we conclude that $M[A ; I, J ; P]$ is defined by a finite complete presentation.

The existence of an entry in the matrix for which the variant semigroup is isomorphic to $A$ is an important condition of the theorem. A related idea to that of variant semigroups is that of mid-monoids. An element $u$ in a semigroup $A$ is called a mididentity if $a u b=a b$, for all $a, b \in A$. If such $u$ exists in $A$ then $A$ is called a mid-monoid. It is known [10, Lemma 3.6] that a semigroup $A$ is a mid-monoid if and only if $A$ has a regular element $x$ (i.e., exists $x^{\prime} \in A$ such that $x x^{\prime} x=x$ ) such that $A(x) \cong A$. This result becomes evident in the case of monoids since for all invertible elements $x$ of the monoid we have $A \cong A(x)$.

Remark 6.2. If $A$ is a monoid and one of the entries of the matrix $P$ is an invertible element then conditions (i), (ii) and (iii) of Theorem 6.1 hold.

Since in a group all elements are invertible we immediately recover the following result originally proved in [7]:

Corollary 6.3. Let $S$ be a completely 0-simple semigroup with finitely many left and right ideals. If every maximal subgroup of $S$ is defined by a finite complete presentation then so is $S$.

We notice that the complete rewriting system obtained in this case is different from the one obtained in [7].

\section{REFERENCES}

[1] D. J. Anick. On the homology of associative algebras. Trans. Amer. Math. Soc., 296(2):641-659, 1986. 
[2] I. M. Araújo. Finite presentability of semigroup constructions. Internat. J. Algebra Comput., 12(1-2):19-31, 2002. International Conference on Geometric and Combinatorial Methods in Group Theory and Semigroup Theory (Lincoln, NE, 2000).

[3] H. Ayık and N. Ruškuc. Generators and relations of Rees matrix semigroups. Proc. Edinb. Math. Soc., II. Ser., 42(3):481-495, 1999.

[4] R. V. Book and F. Otto. String-rewriting systems. Texts and Monographs in Computer Science. Springer-Verlag, New York, 1993.

[5] F. Catino. Factorizable semigroups. Semigroup Forum, 36(2):167-174, 1987.

[6] R. Cremanns and F. Otto. For groups the property of having finite derivation type is equivalent to the homological finiteness condition $\mathrm{FP}_{3}$. J. Symbolic Comput., 22(2):155-177, 1996.

[7] R. Gray and A. Malheiro. Finite complete rewriting systems for regular monids. Submitted, 2010.

[8] R. Gray and A. Malheiro. A monoid is defined by a complete semigroup presentation if and only if it is defined by a complete monoid presentation. Technical report, CAUL, 2010. http://pessoa.fct.unl.pt/ajm/TechRep/CRSforMonoids.pdf.

[9] J. R. J. Groves and G. C. Smith. Soluble groups with a finite rewriting system. Proc. Edinburgh Math. Soc. (2), 36(2):283-288, 1993.

[10] J. B. Hickey. Semigroups under a sandwich operation. Proc. Edinburgh Math. Soc. (2), 26(3):371-382, 1983.

[11] J. M. Howie. Fundamentals of semigroup theory, volume 12 of London Mathematical Society Monographs. New Series. The Clarendon Press Oxford University Press, New York, 1995. Oxford Science Publications.

[12] M. Kambites. Presentations for semigroups and semigroupoids. Int. J. Algebra Comput., 15(2):291-308, 2005.

[13] N. Kuhn and K. Madlener. A method for enumerating cosets of a group presented by a canonical system. In ISSAC '89: Proceedings of the ACM-SIGSAM 1989 international symposium on Symbolic and algebraic computation, pages 338-350, New York, NY, USA, 1989. ACM.

[14] M. Kunze. Zappa products. Acta Math. Hungar., 41(3-4):225-239, 1983.

[15] M. V. Lawson. Rees matrix semigroups over semigroupoids and the structure of a class of abundant semigroups. Acta Sci. Math., 66(3-4):517-540, 2000.

[16] Martin W. Liebeck, Cheryl E. Praeger, and Jan Saxl. Regular subgroups of primitive permutation groups. Mem. Amer. Math. Soc., 203(952):vi+74, 2010.

[17] A. Malheiro. Finite derivation type for Rees matrix semigroups. Theor. Comput. Sci., 355(3):274-290, 2006.

[18] A. Malheiro. On finite semigroup cross-sections and complete rewriting systems. In Zoran Majkic, Michael Sipser, R. Radha, and Daming Wei, editors, TMFCS, pages 59-63. ISRST, 2008.

[19] A. Malheiro. Finite derivation type for large ideals. Semigroup Forum, 78(3):450-485, 2009.

[20] B. H. Neumann. Decompositions of groups. J. London Math. Soc., 10, 1935.

[21] F. Otto. Conjugacy in monoids with a special Church-Rosser presentation is decidable. Semigroup Forum, 29(1-2):223-240, 1984.

[22] S. J. Pride. Low-dimensional homotopy for monoids II. Groups. Glasg. Math. J., 41(1):1-11, 1999. 
[23] S. J. Pride and J. Wang. Rewriting systems, finiteness conditions, and associated functions. In Algorithmic problems in groups and semigroups (Lincoln, NE, 1998), Trends Math., pages 195-216. Birkhäuser Boston, Boston, MA, 2000.

[24] S. J. Pride and J. Wang. Subgroups of finite index in groups with finite complete rewriting systems. Proc. Edinburgh Math. Soc. (2), 43(1):177-183, 2000.

[25] D. Rees. On semi-groups. Proc. Cambridge Philos. Soc., 36:387-400, 1940.

[26] J. A. Séguier. Théorie des groupes finis: Éléments de la théorie des groupes abstraits. Gauthier-Villars, 1904.

[27] C. C. Squier, F. Otto, and Y. Kobayashi. A finiteness condition for rewriting systems. Theoret. Comput. Sci., 131(2):271-294, 1994.

[28] J. Szép. On the structure of groups which can be represented as the product of two subgroups. Acta Sci. Math. Szeged, 12(Leopoldo Fejer et Frederico Riesz LXX annos natis dedicatus, Pars A):57-61, 1950.

[29] Terese. Term rewriting systems, volume 55 of Cambridge Tracts in Theoretical Computer Science. Cambridge University Press, Cambridge, 2003.

[30] J. Wang. Finite derivation type for semi-direct products of monoids. Theoret. Comput. Sci., 191(1-2):219-228, 1998.

[31] Guido Zappa. Sulla costruzione dei gruppi prodotto di due dati sottogruppi permutabili tra loro. In Atti Secondo Congresso Un. Mat. Ital., Bologna, 1940, pages 119-125. Edizioni Cremonense, Rome, 1942.

Centro de Álgebra da Universidade de lisboa, Av. Prof. Gama Pinto 2, 1649-003 Lisboa, Portugal

Current address: Universidade Aberta, R. da Escola Politécnica, 147, 1269-001 Lisboa, Portugal

E-mail address: jaraujo@ptmat.fc.ul.pt

Current address: Departamento de Matemática, Faculdade de Ciências e Tecnologia da UNL, Quinta da Torre, 2829-516 Monte de Caparica, Portugal

E-mail address: malheiro@cii.fc.ul.pt 